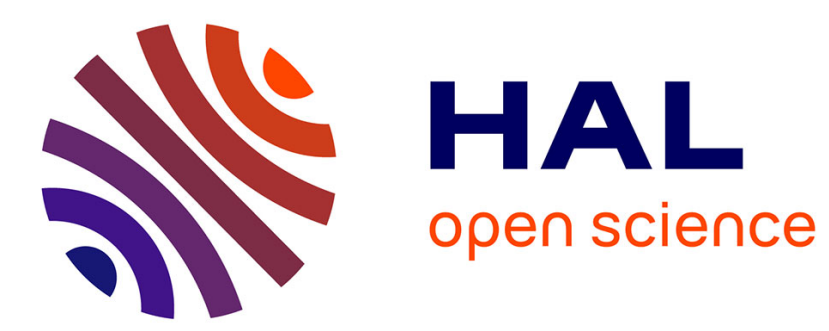

\title{
MÖSSBAUER SPECTROSCOPY OF COMPOUND ES : SUPPORT FOR A Fe IV STATE
}

\author{
G. Lang, K. Spartalian, T. Yonetani
}

\section{To cite this version:}

G. Lang, K. Spartalian, T. Yonetani. MÖSSBAUER SPECTROSCOPY OF COMPOUND ES : SUPPORT FOR A Fe IV STATE. Journal de Physique Colloques, 1976, 37 (C6), pp.C6-217-C6-221. 10.1051/jphyscol:1976645 . jpa-00216755

\section{HAL Id: jpa-00216755 https://hal.science/jpa-00216755}

Submitted on 1 Jan 1976

HAL is a multi-disciplinary open access archive for the deposit and dissemination of scientific research documents, whether they are published or not. The documents may come from teaching and research institutions in France or abroad, or from public or private research centers.
L'archive ouverte pluridisciplinaire HAL, est destinée au dépôt et à la diffusion de documents scientifiques de niveau recherche, publiés ou non, émanant des établissements d'enseignement et de recherche français ou étrangers, des laboratoires publics ou privés. 


\title{
MÖSSBAUER SPECTROSCOPY OF COMPOUND ES : SUPPORT FOR A Fe IV STATE
}

\author{
G. LANG $\left({ }^{*}\right)$, K. SPARTALIAN $(*)$ and T. YONETANI $(* *)$
}

\begin{abstract}
Résumé. - Le composé ES, produit par la réaction de cytochrome c peroxydase et éthyl peroxide, a été étudié par spectroscopie Mössbauer à température et champ magnétique variable. A 4,2 K le déplacement isomérique est $0,05 \mathrm{~mm} / \mathrm{s}(\mathrm{Fe})$; l'éclatement quadrupolaire est indépendant de la température à $1,55 \mathrm{~mm} / \mathrm{s}$, et les largeurs des raies sont égales, approximativement, à la valeur naturelle. L'application d'un champ magnétique à 4,2 $\mathrm{K}$ produit un spectre qui est approximativement celui d'une substance diamagnétique, mais on trouve que le champ magnétique effectif dépend de la température. L'application de la méthode de moindres carrés indique l'existence d'un GCE axial avec $V_{z z}>0$. Le champ magnétique interne est négatif et grand seulement en les directions transversales. Le champ interne est en accord avec une configuration $\mathrm{d}^{4} S=1$ avec $\zeta=400 \mathrm{~cm}^{-1}$ et avec $\mid x z>$ et $|y z\rangle$ supérieur à $\mid x y>$ par $6 \zeta$, environ. La susceptibilité prédite est en accord avec les valeurs publiées. Le modèle prédit que $V_{z z}>0$; mais la grandeur de $\Delta E$ observée indique que sa valeur est diminuée par la charge dans les orbitals de liaison. Il n'y a pas de caractéristique dans le spectre Mössbauer qui correspond au radical libre observable par RPE donc on conclut que ce dernier est loin de Fe.
\end{abstract}

Abstract. - Compound ES, formed by reacting cytochrome c peroxidase with ethyl peroxide, has been examined by Mössbauer spectroscopy over a range of temperature and applied magnetic field. At $4.2 \mathrm{~K}$ the isomer shift is $0.05 \mathrm{~mm} / \mathrm{s}(\mathrm{Fe})$; quadrupole splitting is temperature independent at $1.55 \mathrm{~mm} / \mathrm{s}$, and the linewidth approximates the natural value. Application of a $4 \mathrm{~T}$ field at $4.2 \mathrm{~K}$ produces a spectrum approximating that of a diamagnet, but the effective magnetic field is found to be temperature dependent. Least squares fittings indicate axial efg and magnetic interactions, with $V_{z z}>0$ and a negative internal field which is large only in transverse directions. The internal field is consistent with a d4, $S=1$ configuration with $\zeta=400 \mathrm{~cm}^{-1}$, having $|x z\rangle$ and $|y z\rangle$ lying about $6 \zeta$ above $\mid x y>$. The predicted susceptibility is in reasonable agreement with published values. The model correctly predicts $V_{z z}>0$; the size of the observed $\Delta E$, however, indicates reduction by charge in bonding orbitals. There is no observable Mössbauer spectrum feature corresponding to the EPR-visible free radical, implying that it is remote from the iron.

1. Introduction. - Cytochrome c peroxidase, a heme protein found in yeast $[1,2]$, catalyzes the decomposition of hydroperoxide and oxidizes ferrocytochrome $\mathrm{c}$ to ferricytochrome $\mathrm{c}$ [3]. When reacted with hydroperoxide in absence of cytochrome $\mathrm{c}$ it forms a relatively stable intermediate, Compound $\mathrm{ES}[2,4,5]$. By chemical titration, it is found that Compound ES requires two electrons to reduce it to the native ferric form of the enzyme $[4,5]$. If both were associated with the iron this would imply a formal $\mathrm{V}$ oxidation state. In a somewhat similar way horseradish peroxidase (HRP) combines with hydroperoxide to form Compound I, also two oxidizing equivalents above the ferric native form. Compound I spontaneously converts to Compound II, losing one of its equivalents [6]. Moss [7] has found that the Mössbauer spectra of Compound I and Compound II are very similar, suggesting that both are in the same Fe IV oxidation state. The spectro-

(*) Department of Physics, The Pennsylvania State University, University Park, Pennsylvania 16802, U. S. A.

$(* *)$ Department of Biochemistry and Biophysics, School of Medicine, University of Pennsylvania, Philadelphia, Pennsylvania 19174, U.S. A. photometric similarity between Compound II and Compound ES suggests that these are also similar $[1,4$, 8], and that both Compound $I$ and Compound ES have an oxidizing equivalent not directly associated with the heme iron [9]. Considerable support derives from the observation of a free radical EPR signal in Compound ES [9].

Susceptibility measurements of Compound ES [10] have yielded a fairly constant effective spin in the temperature range $85 \mathrm{~K}$ to $240 \mathrm{~K}$, and have been interpreted in terms of a spin 1 iron and an independent spin $\frac{1}{2}$. In order to test this model and determine the detailed nature of the iron site, we have subjected Compound ES to Mössbauer spectroscopy, a probe capable of distinguishing unambiguously among zero integral, and half-integral spin configurations.

2. Experimental methods. - CCP was purified from bakers' yeast [1] and twice crystallized by dialysis against distilled water [11]. The synthetic enzymes containing ${ }^{57} \mathrm{Fe}$ enriched protohemes were prepared by the chemical exchange of the heme group, as described in detail elsewhere [12]. The samples of synthetic 
enzymes were crystallized by dialysis against distilled water, the crystals were then collected by centrifugation and dissolved in $0.2 \mathrm{M}$ potassium phosphate buffer, $\mathrm{pH}$ 7.0. The enzyme solutions were centrifuged to remove insoluble material and spectrophotometrically assayed to determine the molar concentrations. Conversion to Compound ES was achieved at $23^{\circ} \mathrm{C}$ by adding a slight excess of $\mathrm{C}_{2} \mathrm{H}_{5} \mathrm{OOH}$, and Mössbauer samples were quickly frozen in liquid nitrogen.

The Mössbauer spectrometer was of the constant acceleration type and the source was ${ }^{57} \mathrm{Co}$ in rhodium. In calibrations with thin iron foils [13] linewidths were typically $0.23 \mathrm{~mm} / \mathrm{s}$, FWHM. Linearity of the velocity scale was better than $0.1 \%$, and calibrations were stable to $0.2 \%$ over periods of order 1 week. Measurements in high transverse magnetic fields were obtained using a split-coil superconducting magnet (American Magnetics). For temperatures above $4.2 \mathrm{~K}$ the sample was placed in a small counter-dewar on a heated copper tongue, with silicone grease and aluminum foil to ensure good thermal contact. The temperature, measured with a gold-iron thermocouple, has an estimated uncertainty of $3 \%$.

3. Results. - Mössbauer spectra of Compound ES are shown in figure 1, together with theoretical curves.

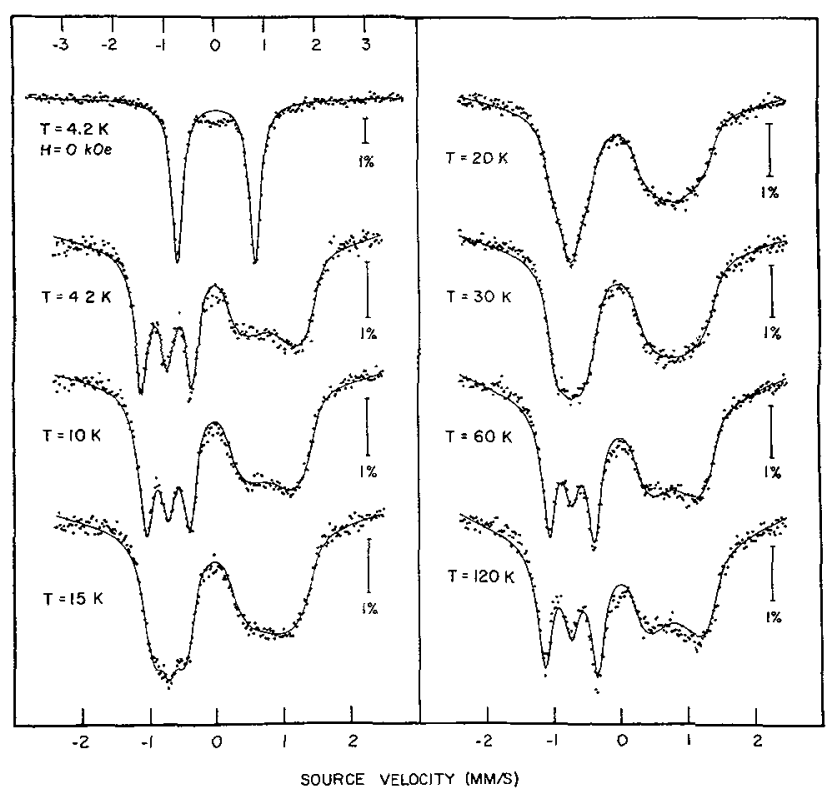

FIG. 1. - Mössbauer spectra of Compound ES at various temperatures in a transverse magnetic field of $4 \mathrm{~T}$. The top velocity scale on the left applies only to the zero-field spectrum immediately below it. The solid lines are calculated, as discussed in the text.

The upper left hand spectrum was observed at $4.2 \mathrm{~K}$ in the absence of applied magnetic field. The main quadrupole pair has isomer shift $0.05 \mathrm{~mm} / \mathrm{s}(\mathrm{Fe})$ and a splitting of $1.55 \mathrm{~mm} / \mathrm{s}$. The linewidth is $0.26 \mathrm{~mm} / \mathrm{s}$, not much above the minimum observed in our spectrometer, so there is no evidence of internal magnetic hyperine interaction. This spectrum remains essentially unchanged at higher temperatures, with the quadrupole splitting remaining constant up to $195 \mathrm{~K}$, the highest temperature of observation. The observed spectrum deviates from the computed pair of Lorentzian lines in the region of zero velocity. This may be evidence of a small amount of high-spin ferric material, an impurity commonly accompanying Compound ES and probably suggesting an incomplete reaction of the native protein. Application of a $4 \mathrm{~T}$ field at $4.2 \mathrm{~K}$ yields a spectrum which is very near to that expected from a diamagnet with positive electric field gradient. The variation produced upon raising the temperature, however, gives evidence of an internal contribution to the total effective magnetic field. In order to quantify this the spectra were fitted [14] using the following assumed form of the hyperfine interaction Hamiltonian :

$$
\begin{aligned}
J e=\frac{Q V_{z z}}{4}\left[I_{z}^{2}-\frac{5}{4}\right. & \left.+\frac{\eta}{3}\left(I_{x}^{2}-I_{y}^{2}\right)\right] \\
& -g_{\mathrm{N}} \beta_{\mathrm{N}} \sum_{i=x, y, z} I_{i} H_{i}\left(1+\omega_{i}\right) .
\end{aligned}
$$

The first term is the quadrupole interaction, and the second is the magnetic hyperfine interaction, incorporating both the direct effect of the applied field and the interaction of the nucleus with the effective internal field of the complex. The latter is represented by the quantities $\omega_{i}$ which are functions of the electronic level scheme and the temperature. The form of the Hamiltonian implies fast relaxation among electronic levels. The simplifying assumption that magnetic and quadrupole interactions have the same principal axis system is justified by the satisfactory fits obtained. The value of $\eta$ was initially found by fitting the $120 \mathrm{~K}$ data, where the internal magnetic field effect was small. The result was that $V_{z z}$ is positive and $\eta=0.02$, which is not significantly different from zero, so an axially symmetric efg was used in the remaining fits. A further tentative assumption, that the magnetic hyperfine interaction was axially symmetric about the same axis, was justified by the very satisfactory fits which resulted. The experimentally determined values of $\omega_{z}$ and $\omega_{\perp}$ are shown in table $\mathrm{I}$, and are plotted in figure 2 , where they

\section{TABLE I}

The internal field coefficients $\omega_{\perp}$ and $\omega_{z}$ obtained by fitting the Mössbauer spectra from Compound ES

\begin{tabular}{rcc}
$T(\mathrm{~K})$ & $\omega_{z}$ & $\omega_{\perp}$ \\
\hline 4.2 & $+\overline{0} .01$ & $-\overline{1.74}$ \\
10.0 & -0.02 & -1.60 \\
15.0 & -0.06 & -1.34 \\
20.0 & -0.08 & -0.96 \\
30.0 & -0.08 & -0.76 \\
60.0 & -0.08 & -0.35 \\
120.0 & -0.04 & -0.18
\end{tabular}




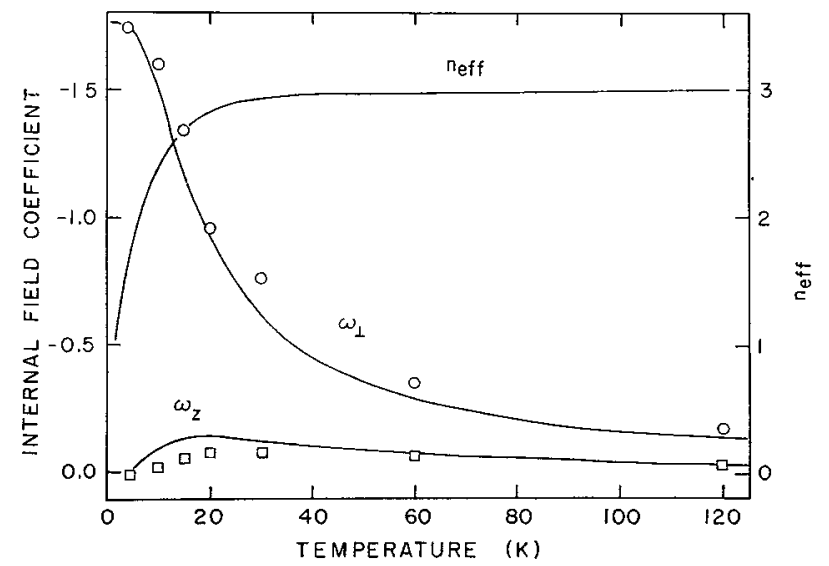

FIG. 2. - Internal field coefficients $\omega_{z}$ and $\omega_{\perp}$ and effective spin number $n_{\text {efr }}$ plotted as a function of temperature. Open circles and squares are experimental points obtained from the fits in figure 1 . The solid lines are obtained from calculations based on the theory discussed in the text.

are compared with predictions based on a model described below. The $\omega_{i}$, being added to unity in eq. (1), are not determined with good percentage accuracy when they are small. Thus the values of $\omega_{z}$ are acceptably close to the calculated curve of figure 2 . It is clear that the $4.2 \mathrm{~K}$ spectrum looks much like that of a diamagnet because in the transverse directions the internal field is such as to produce a total effective field very near in magnitude to the applied field, although reversed in direction. The value $\omega_{\perp}=-2$ would produce this condition exactly; the resulting spectrum would be close (but not identical) to that of a diamagnet.

4. Discussion. - Chemical titrations indicate that the Compound ES molecule must have a half-integral number of electrons, and EPR measurements detect a free radical signal. This material qualifies as magnetically dilute, so it is reasonable to expect slow spin relaxation at liquid helium temperature. If the halfintegral spin were located on the iron we would expect it to produce magnetic hyperfine structure in the zerofield Mössbauer spectrum. This is borne out in all ferric heme proteins which have been investigated [15]. The simple quadrupole splitting and narrow linewidths of the 4.2 K zero-field Mössbauer spectrum of Compound ES put an upper limit to the internal field of a few tenths of a Tesla, to be compared with fields of order 30 to $50 \mathrm{~T}$ seen in ferric heme proteins. Thus if the unpaired electron wave function has some iron orbital character in Compound ES, the absolute square amplitude of this component is not more than a few percent. It is very unlikely that the half-integral spin is located any closer to the iron than a second neighbor position.

Since the magnetic Mössbauer data indicate the presence of integral spin, the possibilities of $\mathrm{d}^{6}$ and $\mathrm{d}^{4}$ iron electronic states must be considered. It is difficult to see how the $\mathrm{d}^{6}$ assignment could be made consistent with the overall oxidation state of the molecule. Further- more, the internal magnetic field is much smaller than in a typical high-spin ferrous material. Finally, the isomer shift of ES is much smaller than that of both high-spin and low-spin ferrous compounds [16], and is in fact significantly lower than that of any known heme protein compound excepting those formed by reaction with peroxide. The iron in HRP peroxide has been interpreted by Moss et al. [7] as Fe(IV) with four 3d electrons. We can see little alternative but that the same description applies in the present case as well.

The sizeable and temperature independent quadrupole splitting of ES indicates the presence of a large crystal field with symmetry lower than cubic. The low symmetry elements of the crystal field will affect also the low temperature susceptibility, so we have the problem of finding a crystal field which accounts for the observed quadrupole splitting, internal magnetic field, and susceptibility, together with their temperature dependence. Kotani [17] has discussed the magnetic properties of the $\mathrm{d}^{4}$ spin 1 complex in a cubic field. The lower symmetry problem also has been treated in detail, and the essential results tabulated separately [18], so we present here only an outline. The model which we consider has a large cubic field, so the $t_{2 g}$ orbitals containing the four electrons, are well separated from the $e_{\mathrm{g}}$. The axial field is specified by $\Delta$, the energy of the degenerate $|x z\rangle,|y z\rangle$ pair relative to $|x y\rangle$. The axial field is assumed to lie along a fourfold cubic field axis, in keeping with the observed coincidence of efg and magnetic axes. The electronic Hamiltonian may be written as

$\mathcal{H}=\sum_{k=1}^{4} V_{\mathrm{ck}}+\sum_{k} \zeta \mathbf{l}_{k} \cdot \mathbf{S}_{k}+2 \beta \mathbf{H} \cdot \sum_{k}\left(\mathbf{l}_{k}+2 \mathbf{S}_{k}\right)$

where the terms represent the axial crystal field, spinorbit coupling and Zeeman interaction with the applied field $\mathbf{H}$. The summations are over four electrons; the energy of mutual electronic repulsion enters implicitly in that only states with $S=1$ are used to form the basis. Setting up and diagonalizing the Hamiltonian (2) is discussed in detail in reference [18].

The magnetic hyperfine interaction is described by the Hamiltonian

$$
\begin{aligned}
& \mathscr{H}_{\mathbf{M}}= P \sum_{k}\left\{\mathbf{L}_{k} \cdot \mathbf{I}+3\left(\hat{r}_{k} \cdot \mathbf{s}_{k}\right)\left(\hat{r}_{k} \cdot \mathbf{I}\right)-\right. \\
&\left.\quad-\left(\mathbf{s}_{k} \cdot \mathbf{I}\right)-\kappa\left(\mathbf{s}_{k} \cdot \mathbf{I}\right)\right\}-g_{\mathrm{N}} \beta_{\mathrm{N}} \mathbf{I} \cdot \mathbf{H} \\
& \equiv-g_{\mathrm{N}} \beta_{\mathrm{N}} \sum_{i} I_{i} H_{i}\left(1+\omega_{i}\right) \\
&\left.P \quad=2 g_{\mathrm{N}} \beta \beta_{\mathrm{N}}<r^{-3}\right\rangle_{\mathrm{effm}}
\end{aligned}
$$

In (3) the orbital, dipolar, and contact hyperfine interactions are added to the direct nuclear interaction with the applied field H. $P$ is taken as $-4.19 \mathrm{~mm} / \mathrm{s}$ $\left({ }^{57 \mathrm{~m}} \mathrm{Fe}\right)$ and $\kappa$ is taken as 0.35 , both typical values for heme proteins $[15,16]$. We are interested in the case of large $\mathbf{H}$, where the applied field determines electronic behavior independent of nuclear coordinates, so we may 
solve eq. (2) and replace the electronic operators in (3) by their expectation values. The interaction with unpaired electrons thus becomes equivalent to an effective field acting on the nucleus, and expression (4) is justified. When more than one electronic state is thermally accessible, we take thermal averages of the expectation values, assuming fast electron spin relaxation. This works in the present case and is consistent with the usual behavior of integral spin iron complexes.

In the first term of eq. (1), $V_{z z}$ and $\eta$ depend on the distribution of all extranuclear charge. Usually the $3 d$ electrons of the iron dominate this, and initially we neglect other contributions. We make the assumption that the delocalization of the $d$ electrons is such that a single electron in an $|x y\rangle$ orbital would give a quadrupole splitting $\Delta E=3 \mathrm{~mm} / \mathrm{s}$, with $V_{z z}$ of course positive $[15,16]$. Investigation of a range of values for the crystal field parameter $\Delta$ indicates that $\Delta \gg \zeta$ or its equivalent in another coordinate system is required to reproduce the observed $\omega_{i}$. To a good approximation, this places one electron in each of $|y z\rangle$ and $|x z\rangle$, and two in $|x y\rangle$, producing a positive and temperature independent efg as required, but with $\Delta E$ near $3 \mathrm{~mm} / \mathrm{s}$, about twice our measured value. It is a general feature of chemical bonding that the charge loss or gain implied in a formal change of oxidation state is to a considerable extent compensated by covalent charge transfer. Thus with a formal IV oxidation state we expect significant back donation of electron charge to the central ion, i. e., the presence of filled bonding orbitals which have significant iron character. In the present case a filled bonding level with $25 \%$ iron $\left|3 z^{2}-r^{2}\right\rangle$ character would be sufficient to reduce the quadrupole interaction by the desired amount.

Assuming a typical value $\zeta=400 \mathrm{~cm}^{-1}$, we have chosen the parameter $\Delta=5.98 \zeta$ to fit as well as possible the induced internal field and its temperature dependence. The predicted $\omega_{\perp}$ and $\omega_{z}$ at $4 \mathrm{~T}$ are shown as solid curves in figure 2 , where they may be compared with the experimental results. A small saturation effect is predicted : in the low field limit the same crystal field value would yield very low-temperature values of $\omega$ about $9 \%$ greater than those shown. Above about $25 \mathrm{~K}$ the difference is negligible.

The susceptibility and the Mössbauer measurements are of course related. In figure 2 we show the predicted value at $4 T$ of $n_{\text {eff }}$, the effective spin number, which is related to the susceptibility by $\chi=N n_{\text {eff }}^{2} \beta^{2} / 3 k T$. Here we have included only that part of the susceptibility associated with spin residing on the iron complex. Again, a slight saturation effect is present : at very low temperature the predicted low field $n_{\text {eff }}$ would be about $4 \%$ higher, with the difference becoming negligible for temperatures over $20 \mathrm{~K}$. Unfortunately we do not have any low temperature susceptibility measurements to compare with our predictions. Iizuka et al. [10], who have measured the susceptibility above $90 \mathrm{~K}$, find the iron contribution equivalent to an $n_{\text {eff }}$ value of about 3.8, compared with our predicted 3.0 to 3.1. If we attempt to explain the discrepancy in terms of a highspin $\left(S=\frac{5}{2}\right)$ component in the susceptibility sample, it would require that about $20 \%$ of the iron be in this form.

The ESR signal of Compound ES, which is isotropic at $77 \mathrm{~K}$, is reduced to axial symmetry at $4.2 \mathrm{~K}$ [19]. It is possible that the spin responsible for it resides in the vicinity of the iron, say as a second neighbor, and overlaps slightly with its unpaired spin. The overlap would be equivalent to an internal field and would modify the apparent $g$ value. Although this need not be always proportional to the internal field sensed in the Mössbauer measurement, it should have roughly the same temperature dependence. Thus a careful study of the ESR signal below $77 \mathrm{~K}$ could cast additional light on the structure of the peroxidase complex.

5. Conclusion. - The combination of Mössbauer and ESR results leave little doubt that the iron of Compound ES retains a unit unpaired electron spin, and experiences a large crystal field splitting of axial symmetry.

Any description of this complex as ferric is out of the question. A model based on a d ${ }^{4}$ assignment and large axial crystal field is consistent with both the susceptibility and the magnetic features of the Mössbauer spectra. The model also reproduces the sign of the quadrupole interaction; its magnitude requires the assumption of covalent electron donation to an extent consistent with the behavior observed in other heme compounds.

Acknowledgements. - This work has been carried out at The Pennsylvania State University under NIH Grant (HL-16860-02) from the National Heart and Lung Institute. Work at the University of Pennsylvania has been carried out under NIH Grant (HL-14508) from the National Heart and Lung Institute and Grant (BMS-73-00970) from the National Science Foundation.

\section{References}

[1] Yonetani, T. and RAY, G. S., J. Biol. Chem. 240 (1965) 5403-5408.

[2] Altschul, A. M., Abrams, R. and Hogness, T. R., J. Biol. Chem. 136 (1940) 777-794.

[3] Yonetani, T. and RaY, G. S., J. Biol. Chem. 241 (1966) 700706.
[4] Yonbtani, T., J. Biol. Chem. 240 (1965) 4509-4514.

[5] Yonetani, T., J. Biol. Chem. 241 (1966) 2562-2571.

[6] George, P., Biochem. J. 54 (1953) 267-276.

[7] Moss, T. H., Ehrenberg, A. and Bearden, A. J., Biochemistry 8 (1969) 4159-4162.

[8] Chance, B., Arch. Biochem. Biophys. 41 (1952) 404-415. 
[9] Yonetani, T., Schleyer, H. and Ehrenberg, A., J. Biol. Chem. 241 (1966) 3240-3242.

[10] Irzuka, T., Kotani, M. and Yonetani, T., Biochim. Biophys. Acta 167 (1968) 257-267.

[11] Yonetan,, T., Chance, B. and Kajwwara, S., J. Biol. Chem. 241 (1966) 2981-2982.

[12] Yonetani, T., Asakura, T., J. Biol. Chem. 243 (1968) 47154721.

[13] Preston, R. S., Hanna, S. S. and Heberle, J., Phys. Rev. 128 (1962) 2207-2218.
[14] LANG, G. and DaLe, B., Nucl. Instr. Methods 116 (1974) 567-571.

[15] LANG, G., Q. Rev. Biophys. 3 (1970) 1-60.

[16] Lang, G, and Marshall, W., Proc. Phys. Soc. 87 (1966) 3-34.

[17] Kotani, M., J. Phys. Soc. Japan 4 (1949) 293-297.

[18] Oosterhuis, W. T. and Lang, G., J. Chem. Phys. 58 (1973) 4757-4765.

[19] Yonetani, T. (unpublished). 